\title{
P-1116
}

(105)

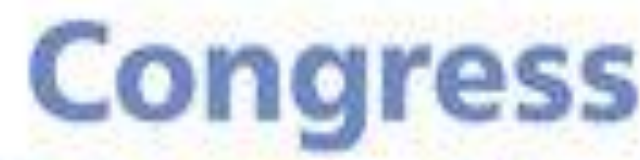
2017

4-8 December Abu Dhabi

\section{Psychometric properties of Diabetes Self-Management Questionnaire (DSMQ) in Urdu}

Allah Buksh 1,2 , Shaun Wen Huey Lee, ${ }_{1}$ Priyia Pusparajah ${ }_{3}$, Andreas Schmitt4, Tahir Mehmood Khan 1

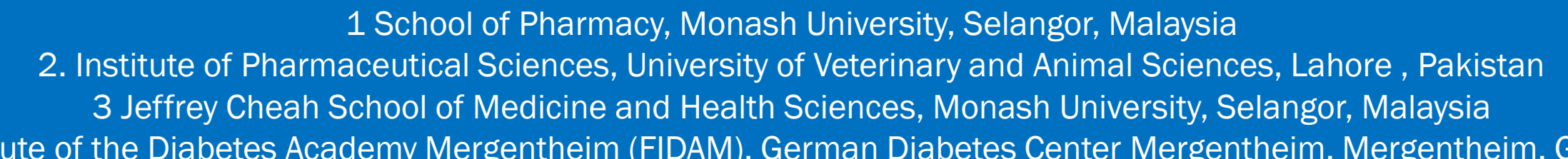

\section{INTRODUCTION}

Globally 415 million people have been diagnosed with diabetes, and the count is predicted to reach 642 million by 2040.(1)

- Pakistan is ranked 7 th for diabetes disease burden in the world, with prevalence rate of $11 \%$ in 2011, and it is anticipated to reach 15\% (14 million) by year 2030. If the present scenario continues, Pakistan is expected to move to top 4th place. (2)

- Self-management activities, like, healthy diet, regular exercise, selfmonitoring of blood glucose and rational use of medication, are considered to play the key role in establishing euglycaemia. ${ }^{(3)}$

- Assessment of patients' self-management activities helps in identifying the reasons for poor glycaemic control. For this reason a standardised self-care assessment tool could be of a great value for researchers and clinicians seeking to evaluate multiple domains of diabetes patients' self-care. ${ }^{(4)}$

- The aim of study was to illustrates the translation process and psychometric evaluation of DSMQ's in Pakistani people with type 2 diabetes.

\section{METHODOLOGY}

\section{- Design: Cross-sectional survey}

- Subjects: adult age (>30 years), type 2 diabetes, diagnosed at least one year before, recent HbA1c lab test (not more than 8 weeks older from the date of interview), taking hypoglycemic medications and sufficient communication skills in the Urdu language.

- Sample size: A target sample size of 160 patients with type 2 diabetes was estimated based on the number of items to participant ratio of $1: 10 .{ }^{(5)}$

- Study instrument: Urdu version of 16-item DSMQ(5) was used. The survey took approximately 10-15 minutes to be completed.

- Instrument translation: The English version of the DSMQ was translated into Urdu language using a standardised forward and backward translation procedure, as recommended by Bradley.(6)

- Scoring Criteria: The scoring of the DSMQ involves summing up the scores of all items after reversing the scores of nine negatively keyed statements (so that higher scores represent more effective self-care). The scale scores are then transformed to a scale ranging from 0 to 10 , where a score of 10 indicates the most effective self-care behaviour.

- Ethics: MUHREC (Approval No.7767)

- Data Analysis: Statistical Package for Social Science version $21 \AA$ and AMOS version 21.0.0 were used to analyse the data. Both descriptive and inferential statistics were applied to attain the objectives of the study.

\section{CORRESPONDING AUTHOR}

\section{Allah Bukhsh}

School of Pharmacy, Monash University Malaysia

Email : allah.bukhsh@monash.edu

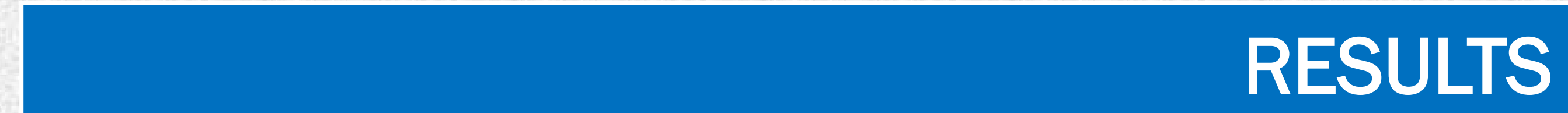

The total sample consisted of 130 patients (response rate $=81.25 \%$ ) with type 2 diabetes.
Table 1. Characteristics of respondents $(n=130)$

\begin{tabular}{|c|c|}
\hline Characteristics & $\mathrm{n}(\%)$ or mean $\pm \mathrm{SD}$ \\
\hline Mean age $( \pm S D)$ & $51.3 \pm 10.4$ \\
\hline Female gender (\%) & $57.6 \%$ \\
\hline $\mathrm{BMI}(\mathrm{kg} / \mathrm{m} 2)$ & $29.7 \pm 6.2$ \\
\hline Diabetes duration (years) & $8.5 \pm 7.0$ \\
\hline HbA1c value (\%) & $8.6 \pm 1.9$ \\
\hline \multicolumn{2}{|l|}{ Anti-diabetic therapy } \\
\hline Exclusively insulin & $14(10.8)$ \\
\hline Insulin combined with oral hypoglycemic agents & 57 (43.9) \\
\hline Oral hypoglycemic agents only & 59 (45.3) \\
\hline
\end{tabular}

The mean $( \pm S D)$ inter-item correlation was 0.76 ( \pm 0.09). The overall Cronbach's $\alpha$ for the Urdu version of DSMQ scale was 0.96 . The Spearman correlation between HbA1c and DSMQ sum scale was $-0.78(p<0.001)$.

Table 2. Comparison of the DSMQ self-care activities in patients with $\mathrm{HbA} 1 \mathrm{c} \leq 7.5 \%$, from 7.6 to $8.9 \%$ and $\geq 9.0 \%$

\begin{tabular}{|c|c|c|c|c|c|c|c|}
\hline DSMQ & $\begin{array}{c}H b A 1 c \leq 7.5 \% \\
(n=49)\end{array}$ & Sign..$^{a}$ & $\begin{array}{c}\text { HbA1c } 7.6-8.9 \% \\
(n=35)\end{array}$ & Sign. ${ }^{b}$ & $\begin{array}{c}\text { HbA } 1 c \geq 9.0 \% \\
(n=46)\end{array}$ & Sign. ${ }^{\mathrm{c}}$ & $\begin{array}{l}\text { ANOVA } \\
\text { P-value }\end{array}$ \\
\hline Glucose Management & $8.18 \pm 1.46$ & $\ddagger$ & $4.04 \pm 1.71$ & ns & $3.23 \pm 2.22$ & $\ddagger$ & $<0.001$ \\
\hline Dietary Control & $7.72 \pm 1.30$ & $\ddagger$ & $3.62 \pm 1.88$ & * & $2.74 \pm 1.55$ & $\ddagger$ & $<0.001$ \\
\hline Physical Activity & $6.96 \pm 1.97$ & $\ddagger$ & $2.79 \pm 2.35$ & ns & $1.81 \pm 1.86$ & $\ddagger$ & $<0.001$ \\
\hline Health-Care Use & $7.14 \pm 1.62$ & $\ddagger$ & $4.03 \pm 2.22$ & ns & $3.45 \pm 1.99$ & $\ddagger$ & $<0.001$ \\
\hline Sum Scale & $7.64 \pm 1.16$ & $\ddagger$ & $3.63 \pm 1.74$ & ns & $2.81 \pm 1.58$ & $\ddagger$ & $<0.001$ \\
\hline
\end{tabular}

\section{Psychometric properties of the Urdu version of DSMQ}

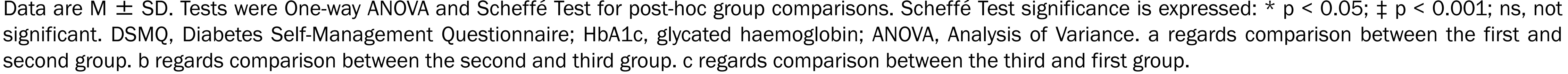

Linear regression analysis revealed that only Glucose Management and Dietary Control were significantly associated with lower HbA1c values $(\mathrm{OR}=-0.42, \mathrm{p}=0.004$ and $=-0.30, p=0.028$, respectively), whereas, Physical Activity and Health-Care Use were not ( $p>0.05)$.

Adequate fit to the data was achieved for single factor model after successively modelling all significant correlations between the items' error terms, with Chi2 = 106.6, $\mathrm{df}=84, \mathrm{p}=0.049$; TLI = $0.98, \mathrm{CFI}=0.99$ and RMSEA $=0.05(90 \% \mathrm{Cl} 0.01-0.07)$. Whereas, a comparatively lower fit indices to data were observed in case of four factor model.

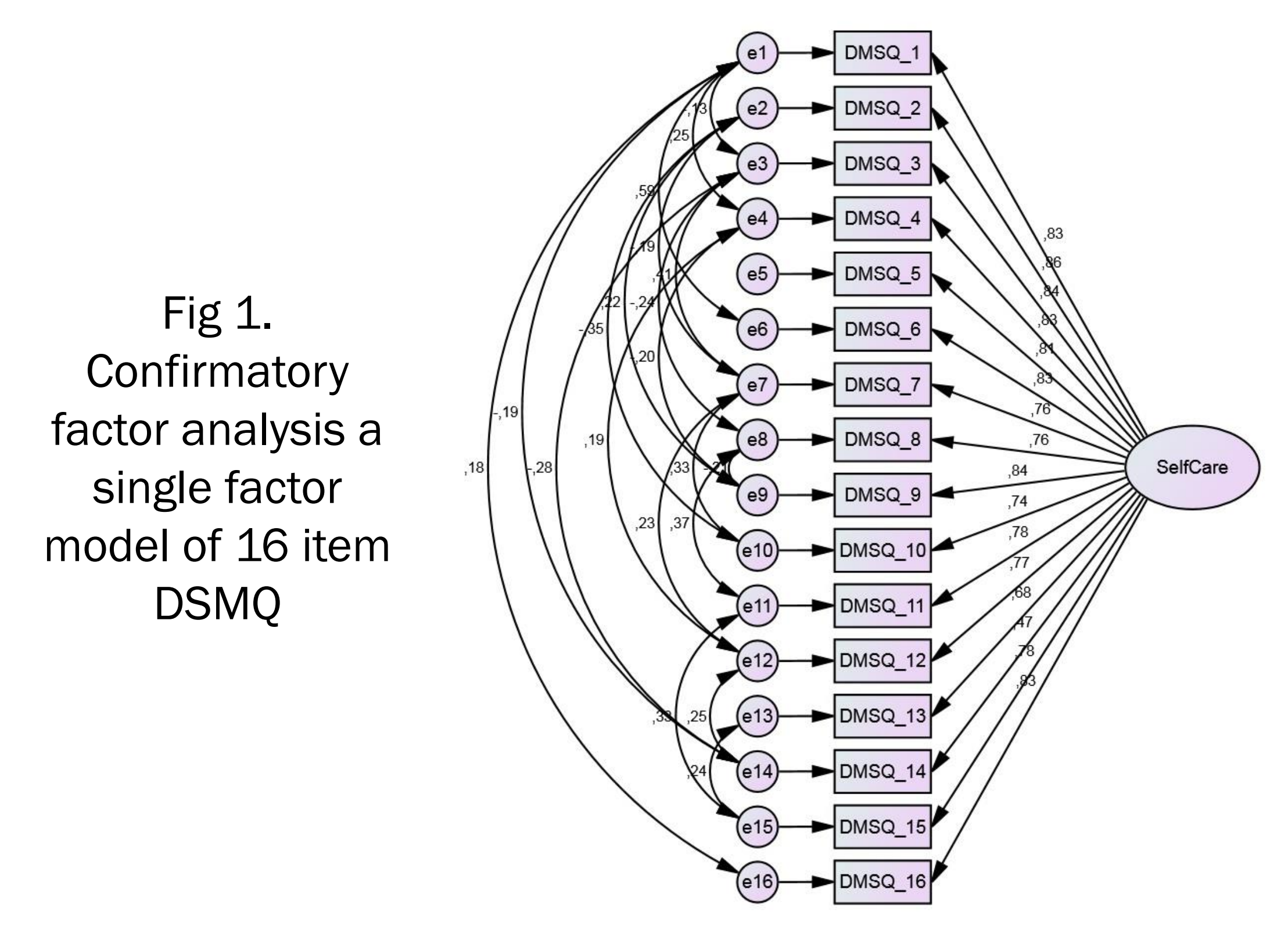

$$
\begin{gathered}
\text { Fig } 2 \\
\text { Confirmatory } \\
\text { factor analysis a } \\
\text { four factor } \\
\text { model of } 16 \\
\text { item DSMQ }
\end{gathered}
$$

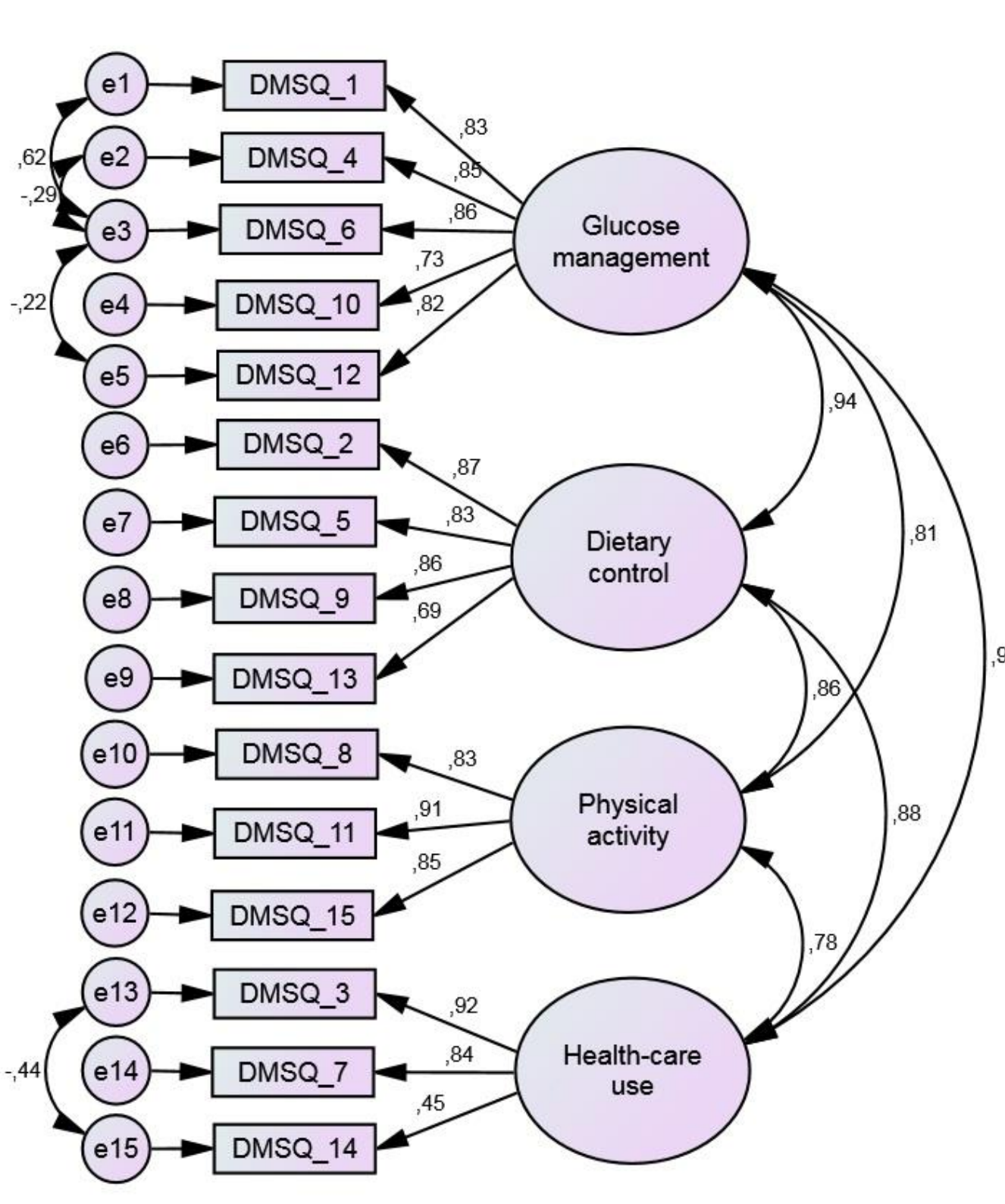

\section{CONCLUSIONS}

- The DSMQ-Urdu version was found to be a valid and reliable tool for measuring self-care activities in type 2 diabetes patients in Pakistan.

- The Urdu version of DSMQ will be of significant value for researchers evaluating the relationship of self-management with glycaemic control and for clinicians seeking to identify their patients' self-care practices requiring improvement.

\section{REFERENCES}

DF DIABETES ATLAS [http://www diabetesatlas.org/.

Bahadar H, Mosta

Disord. 2014;13:1.

Ahola A, Groop PH. Barriers to self-management of diabetes. Diabet Med.2013;30:413-20.

Goodall TA, Halford WK. Self-management of diabetes mellitus: a critical review. Health Psychol. 1991;10:1 Lai PS, Chua SS, Tan CH, Chan SP. Validation of the diabetes, hypertension and hyperlipidemia (DHL) knowledge instrument in Malaysia. BMC Med
Res Methodol. 2012;12:18. Bradley C. Translation of questionnaires for use in different languages and cultures. Handbook of Psychology and Diabetes: a guide to psychological 